\title{
A SPECIFIC METHODOLOGY FOR ATMOSPHERIC EFFECT REDUCTION ON SAR INTERFEROGRAMS
}

\author{
R. Abdelfattah ${ }^{1 ; 2}$, K. Chokmani ${ }^{3}$ and N. Chaabane $e^{1 ; 3}$ \\ ${ }^{1}$ URISA, École Supérieure des Communications, Tunis, Route de Raouède Km 3.5, 2083, Tunisie . \\ 2 Département ITI, Telecom Bretagne, Institut TELECOM, CS 83818 - 29238 Brest Cedex 3 - France \\ ${ }^{3}$ INRS-ETE, 490, rue de la Couronne Québec (Qc), Canada G1K 9A9
}

\begin{abstract}
Interferometric Synthetic Aperture Radar (InSAR) measurements are often biased due to atmospheric effects. Especially, the tropospheric water vapor engenders a delay of SAR signal propagation. In this paper, we propose a specific methodology for atmospheric effects correction on SAR interferograms. It is based on ancillary data collected from NOAA-AVHRR sensor. The specificity of the approach consists in its applicability where no ground truth GPS measurements are available neither for calibration nor for result validation. An adaptive validation demarche is also proposed.
\end{abstract}

Index Terms- Interferometric SAR, atmospheric effects, atmospheric correction, SAR interferograms, NOAAAVHRR.

\section{INTRODUCTION}

More than $35 \%$ of Southern Tunisia (pre-Saharan region, North Africa) is estimated to be at risk or at great risk of desertification. Monitoring the vegetation cover reduction in this region is therefore indispensable for combating and management of the desertification effects. Desertification monitoring needs to be frequent and periodic and this could not be possible with traditional ground surveys. Radar interferometry (InSAR) has proved its utility in the monitoring of desertification. However, the accuracy of the InSAR measurements is strongly conditioned with phase propagation delays through the atmosphere. In general, it is the tropospheric variations that can lead to misinterpretation of InSAR results [1].

The territory covered by this study is located in a coastal area in southern Tunisia, which is under maritime influence. Thus, atmospheric effects, particularly those resulting from the presence of water vapor result in a delay in the radar signal. This leads to an inaccuracy in DEMs generated by In-SAR and therefore to uncertainties in the change detection in the desert extent that could be measured through a time series of InSAR DEMs. Various methods were developed for mitigating the atmospheric effects. It has been shown that methods based on ancillary data (such as GPS observations, ground meteorological data, and optic satellite data) are very effective and reduce the atmospheric effects by about $20-40$ percents.

In this paper, we propose a specific methodology for tropospheric effects correction on SAR interferograms generated over the Southern Tunisia. It is based on ancillary data collected from NOAA-AVHRR sensor. The specificity of the approach consists in its applicability where no ground truth GPS measurements are available neither for calibration nor for result validation. An adaptive validation demarche is also proposed.

\section{TEST SITE AND RELATED PROBLEMATIC}

The study area covers some $22000 \mathrm{Km}^{2}$ and lies between coordinates $\left[34^{\circ} \pm 00^{\prime}, 11^{\circ} \pm 15^{\prime}\right]$ and $\left[32^{\circ} \pm 00^{\prime}, 8^{\circ} \pm 10^{\prime}\right]$ (Fig. 1). In the studied area, we can distinct four physiographic zones with considerable diversity of soils: Coastal plains, mountainous areas, large Depressions or "Chotts" and the Desert zone. In terms of precipitation, the Tunisian south can be divided into three climatic zones following the annual average rainfall quantities which are the semiarid, arid and desert zones. The selected test site corresponds to

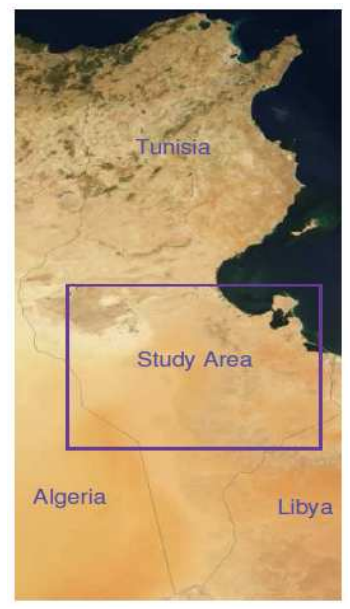

Fig. 1: Study area a costal region with high temperature degrees most of the time. Thus, the evapotranspiration process would play a great role in increasing the volume of the water vapor on the tropospheric related space. It is understood ([2]) that water vapor has the most significant effect on InSAR products. Moreover, in [3] it is demonstrated that atmospheric water vapor effects greater than $4 \mathrm{~cm}$ can be observed even in desert regions. Then, removes the water vapor effects on 
SAR interferogram is imperative in order to improve the accuracy of InSAR measurements.

Several techniques are currently available for monitoring atmospheric water vapor [4]. However, there are no current or planned global sources of observational measurements of atmospheric water vapor at appropriate temporal and spatial sampling scales to satisfy all the various needs [4]. Indeed, existing water vapor effects correction models need a good temporal and spatial distribution of observations. Considering that over the selected test site, the water vapor distribution can be highly variable, existing correction models will not be accurate.

\section{TROPOSPHERIC EFFECTS ON SAR INTERFEROGRAM}

The interferogram measures the phase difference between two SAR images (modulo $2 \pi$ ) which is directly related to the path length difference $\delta D$, in the case of a propagation medium with a refractive index equal to 1 , as follows:

$$
\phi=k_{0} \cdot \delta R
$$

where $k_{0}=2 \pi / \lambda_{s}$ is the wavenumber, $\delta \mathrm{R}=2 \delta \mathrm{D}$ (for repeat pass) and, is the radar signal wavelength. Thus, using a given InSAR formulation [5], (1) will lead to a topographic map.

In the case of a propagation medium unequal to 1 , an incremental path length will be observed due to the signal delay in the medium [6], and then, the new expression of the interferometric phase is given by [2]:

$$
\phi=\frac{4 \pi}{\lambda}\left(\delta D+\Delta_{p} D\right)
$$

where $\Delta_{p} D$ is the extra path length difference induced by the inhomogeneities of the atmosphere. The resultant extra phase would induce a mismeasurement in the interferometric parameter (topographic or surface deformation) estimation. In order to mitigate the atmospheric effects on the InSAR phase we have to determine the variation of the phase error with respect to the atmosphere parameter. However, the major amount of the atmospheric general errors on InSAR images is caused by phase fluctuations due to troposphere $[1,7]$. It is defined as a sum of two component parts: an hydrostatic (or dry) part $\Delta_{p} D_{h}$, depending on $P(\mathrm{mB})$ the total pressure and $T(\mathrm{~K})$ the temperature and a wet part $\Delta_{p} D_{\mathrm{w}}$, depending on $e$ the partial water vapour pressure. $e$ is derived from the relative humidity (\%) [6]. According to the following assumption: the troposphere is an ideal gas in hydrostatic equilibrium, $e$ decreases exponentially and $T$ decreases linearly with elevation, Saastamoinen (1972) proposed the following expressions for $\Delta_{p} D_{h}$ and $\Delta_{p} D_{\mathrm{w}}$ [8]:

$$
\begin{aligned}
& \Delta_{p} D=\Delta_{p} D_{h}+\Delta_{p} D_{w} \\
& \Delta_{p} D=2.277 .10^{-3} \frac{P+\left(0.05+\frac{1225}{T+273.15}\right) e}{1-0.00266 \cos (2 \varphi)-0.00028 \mathrm{~h}}
\end{aligned}
$$

where, $P$ is the total surface pressure in mbar, $e$ the partial water surface pressure in mbar, $T$ the surface temperature in degrees Kelvin and $\varphi$ the geodesic latitude. The results are given in meters.

However, in [2] it is shown that the artefact in the InSAR phase result mainly from the atmospheric (tropospheric) water vapour. The effects caused by the hydrostatic term (variations in pressure and temperature) are not significant compared with those of water vapour [2].

\section{THE PROPOSED METHODOLOGY}

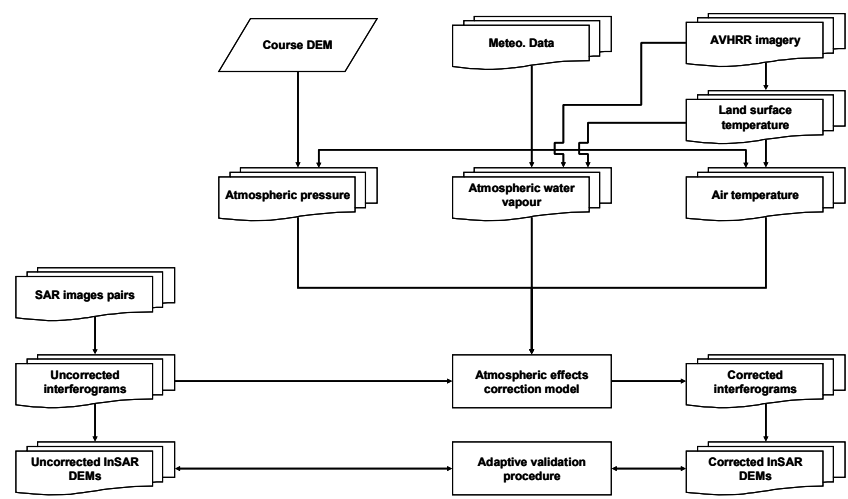

Fig. 2 : The proposed atmospheric effects correction methodology

The atmospheric effects correction methodology is based on the Saastamoinen model. This model which is actually a simplified physical model [8] and uses ancillary data has been applied successfully by several researchers and in different contexts $[6,9]$. As shown by eq. 4 , the delay in the radar signal is estimated using three input meteorological variables: $P, e$ and $T$. Generally, these inputs are available from local observations at meteorological stations or from radiosondes. However, the spatially and temporally discrete nature of these observations and their non-optimal spatial distribution and density prevent from getting a spatially continuous correction over the whole study area.

As illustrated by Fig. 2 we propose an approach that uses satellite AVHRR imagery; ground based meteorological observations and medium resolution DEM (1 $\mathrm{km}$ spatial resolution) to generate spatially continuous input data for the Saastamoinen model throughout the area covered by the interferograms. The proposed methodology was developed and tests over eight (8) interferogram pairs generated using six (6) ERS-1 SAR images acquired in 1996 (Jan. 01, Feb. 05 and Feb. 06) and 1999 (Apr. 06, May 11 
and Jul. 07). SAR images are acquired at 9:00AM local time. Six (6) AVHRR scenes over the study area were used (their acquisition time were selected to be the closest to those of SAR images) as well as standard local observations

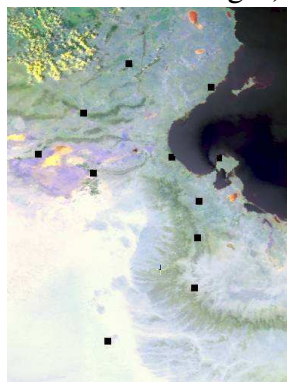

Fig. 3: Location of the used meteorological stations from 11 meteorological stations, located in southern Tunisia (Fig. $3)$, to generate ancillary input variables.

Air temperature maps were produced by means of a polynomial function model calibrated using air temperature ground observations and correspondent Land Surface Temperature (LST) estimated from AVHRR imagery. The Becker and $\mathrm{Li}$ [10] algorithm was employed for LST estimation using Sobrino et al. [11] method for spectral land surface emissivity retrieval. Water vapour pressure was estimated over the study area applying the Choudhury [12] model that relates ground level humidity to precipitable water. This later was extracted from AVHRR channel 4 and 5 brightness temperature difference and LST using a modified version of the algorithm proposed by Mottel et al. [13]. As for ground level atmospheric pressure, a simple exponential model was built relating terrain altitude given by the medium resolution DEM and atmospheric pressure measurements at meteorological stations. All ancillary data estimation procedures were validated with cross-validation technique using the corresponding meteorological ground based observations.

The three ancillary data calculated for each SAR acquisition were resampled at the SAR image spatial resolution and used to estimate the correspondent atmospheric delay using eq 4 . These delays were subtracted from the interferometric delay in order to obtain the corrected interferogram.

Since there is a total lack of in situ GPS measurements for validation purpose, an adaptive validation procedure was applied. This consisted in calculating InSAR terrain elevation variances from uncorrected and corrected for atmospheric effects InSAR DEMs; and quantifying magnitude of InSAR elevation variance decrease or increase between the two types of DEMs using the following formula:

$$
V C R=\frac{V_{c}-V_{u}}{V_{u}}
$$

where $V C R$ is the variance change rate expressed in $\%, V_{c}$ is the corrected InSAR DEMs variance and $V_{u}$ is the uncorrected InSAR DEMs variance.

\section{RESULTS}

Cross-validation results for ancillary data estimation were very satisfactory. Precipitable water was estimation with $\mathrm{R}^{2}$ of 0.77 and RMSE of $4.9 \mathrm{~mm}$. Air temperature was calculated from satellite LST measurements with $\mathrm{R}^{2}$ of 0.89 and RMSE of $2.4 \mathrm{~K}$. As for atmospheric pressure, it was estimated with $\mathrm{R}^{2}$ of 0.94 and RMSE of $3.6 \mathrm{mBar}$.

Here are presented only results for the inteferometric pair April $6^{\text {th }}-$ May $11^{\text {th }} 1999$. Fig. 4 shows the Insar DEMs produced using uncorrected and corrected correspondent interferograms. The corrected DEM is less noisy than the uncorrected one. The elevation range in the corrected DEM is slightly narrower and therefore seems to be more smoothed.

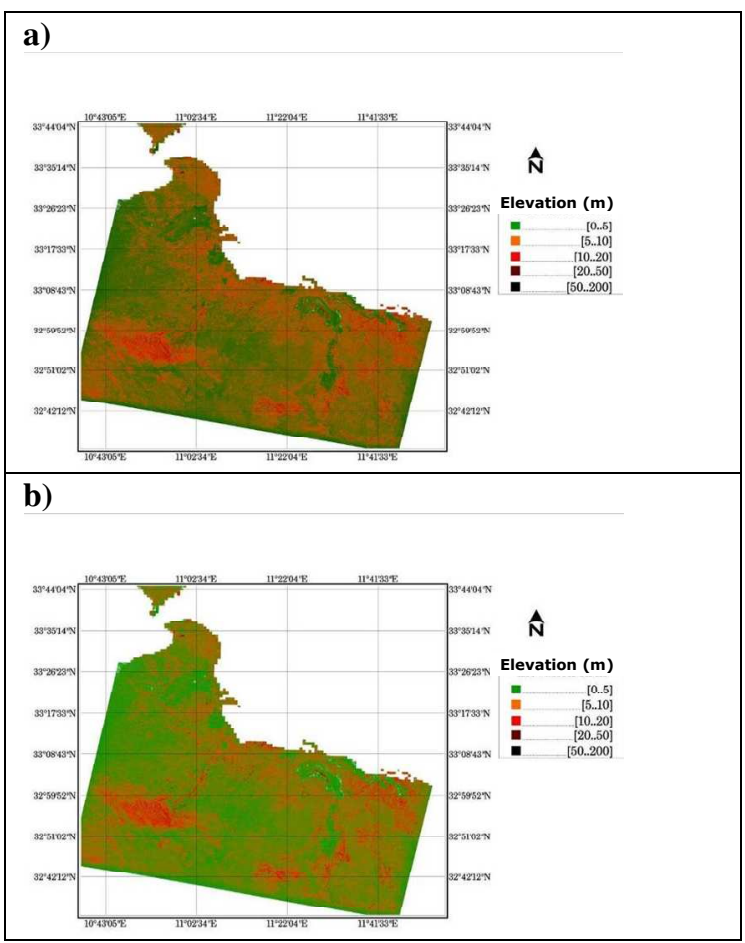

Fig. 4: InSAR DEM for the interferogram pair April $6^{\text {th }}$ - May 11 ${ }^{\text {th }}$, 1999: a) uncorrected for atmospheric effects; b) corrected for atmospheric effects

Fig. 5 presents the map and the histogram of the $V C R$ parameter calculated from all InSAR DEMs processed in this study. As it can be seen from this figure, the proposed correction methodology contributes to reduce significantly the variance in DEM calculation compared to those obtained without atmospheric correction. Indeed, the variance decreased in about $90 \%$ of the territory. The reduction was in average about $20 \%$. As expected, the coastal zone in general has been affected by the largest reduction. 


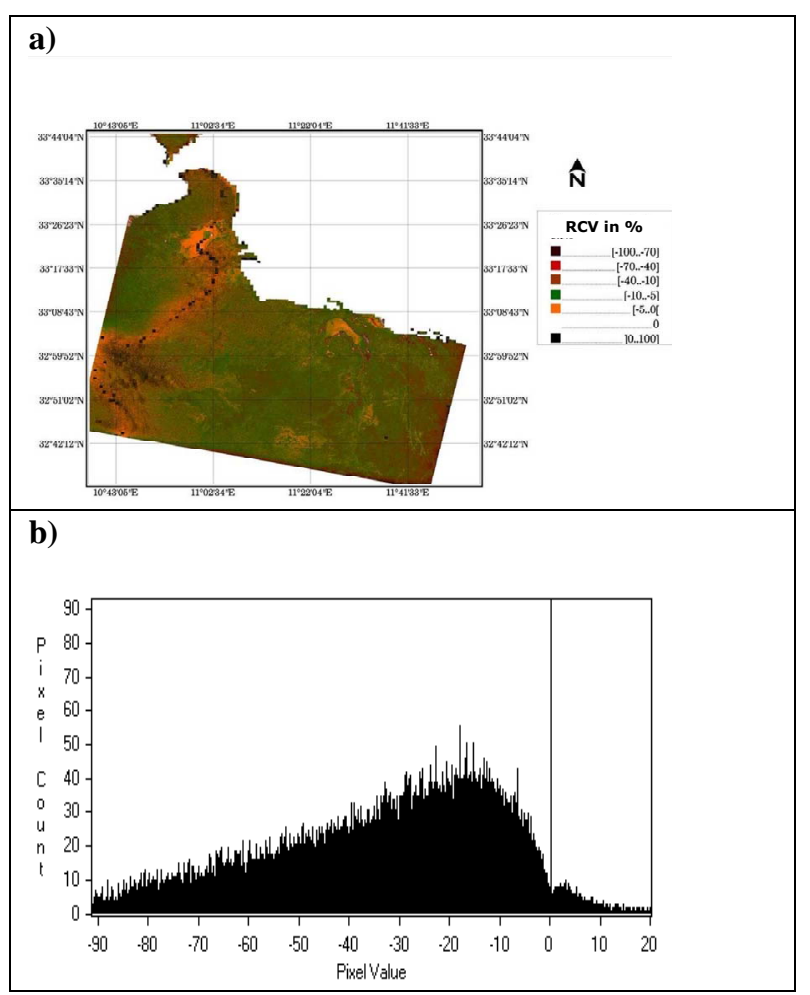

Fig. 5: Change rate in InSAR DEMs variance (VCR) for the interferogram pair April $6^{\text {th }}-$ May 11 ${ }^{\text {th }}, 1999:$ a) map; b) histogram

\section{CONCLUSIONS}

In this study, we propose an atmospheric effects correction approach using ancillary data: AVHRR imagery; ground based meteorological observations and medium resolution DEM $(1 \mathrm{~km})$ to generate spatially continuous input data for the Saastamoinen model. All ancillary data estimation procedures were validated using corss-validation and results were very satisfactory. The proposed correction methodology was developed and tests using ERS-1 SAR images. Results showed that the proposed correction approach reduces the DEMs variance in over than $85 \%$ of the study territory

\section{ACKNOWLEDGMENTS}

The authors would like to acknowledge Agence Universitaire de la Francophonie for the financial support.

\section{REFERENCES}

[1] V. Janssen, L. Ge, and C. Rizos, "Tropospheric corrections to SAR interferometry from GPS observations," GPS Solutions, vol. 8, pp. 140-151, 2004.
[2] H. A. Zebker, P. A. Rosen, and S. Hensley, "Atmospheric effects in interferometric synthetic aperture radar surface deformation and topographic maps," Journal of Geophysical Research B: Solid Earth, vol. 102, pp. 7547-7563, 1997.

[3] Z. Li, "Reduction of atmospheric water vapour effects on ENVISAT ASAR interferograms using MERIS near IR measurements " in The XXI Congress of the International Society for Photogrammetry and Remote Sensing Beijing, China, 2008.

[4] P. W. Webley, R. M. Bingley, A. H. Dodson, G. Wadge, S. J. Waugh, and I. N. James, "Atmospheric water vapour correction to InSAR surface motion measurements on mountains: Results from a dense GPS network on Mount Etna," Physics and Chemistry of the Earth, vol. 27, pp. 363-370, 2002.

[5] R. Abdelfattah and J. M. Nicolas, "Topographic SAR interferometry formulation for high-precision DEM generation," IEEE Transactions on Geoscience and Remote Sensing, vol. 40, pp. 2415-2426, 2002.

[6] R. F. Hanssen and A. Feijt, "A first quantative evaluation of atmospheric effects on SAR interferometry.," in Proceedings of the Fringe 96 Workshop, Zurich, Switzerland, 1996.

[7] R. F. Hanssen, Radar Interferometry: Data interpretation and Error Analysis. Dordrecht: Kluwer Academic Publishers, 2001.

[8] J. Saastamoinen, "Atmospheric correction for troposphere and stratosphere in radio ranging of satellites," in The use of artificial satellites for Geodesy, 1972, pp. 247-251.

[9] W.-J. Qu, W.-Y. Zhu, S.-L. Song, and J.-S. Ping, "Evaluation of the Precision of Three Tropospheric Delay Correction Models," Chinese Astronomy and Astrophysics, vol. 32 pp. 429-438, 2008.

[10] F. Becker and Z. L. Li, "Towards a local split window method over land surfaces," International journal of Remote Sensing, vol. 11, pp. 369-393, 1990.

[11] J. A. Sobrino, N. Raissouni, and Z.-L. Li, "A comparative study of land surface emissivity retrieval from NOAA data.," Remote Sensing of Environment, vol. 75, pp. 256-266, 2001.

[12] B. J. Choudhury, "Comparison of models relating precipitable water to surface humidity using globally distributed radiosonde data over land surfaces," International Journal of Climatology,, vol. 16, pp. 663-675, 1996.

[13] C. Motell, J. Porter, J. Foster, M. Bevis, and S. Businger, "Comparison of precipitable water over Hawaii using AVHRRbased split-window techniques, GPS and radiosondes," International Journal of Remote Sensing, vol. 23 pp. 2335-2339, 2002. 